\title{
Revue des livres
}

Le risque radioactif. Devenir des radionucléides dans l'environnement et impacts sur la santé, Jean-Claude AMIARD, Tec\&Doc Lavoisier, 2013, ISBN : 978-2-7430-1495-

7, 627 pages, $135 €$.

Jean Claude Amiard est très prolixe, après Les risques chimiques environnementaux (2011), il publie aujourd'hui un ouvrage sur le risque radioactif. Tout comme le précédent, ce livre est très complet et n'hésite pas à redéfinir les connaissances de base, revoir toutes les définitions pour chaque sujet abordé. Ceci est vrai pour la radioactivité, la physique nucléaire, l'organisation du monde vivant, etc. Bref J.C. Amiard part toujours du plus fondamental pour en arriver au risque radioactif qu'il décrit. Ceci explique en partie pourquoi ce livre est si copieux. Si le lecteur s'impatiente, il peut toujours zapper.

La documentation est très complète et ne se limite pas aux dernières apparitions sur Internet. L'auteur remonte le plus souvent (pas toujours) à la source de l'information ou de l'histoire. Est-ce toujours utile ? Je ne sais pas, le lecteur est-il aujourd'hui intéressé par les réserves mondiales d'uranium pays par pays en 2006 ? De telles informations sont mieux actualisées dans les documents de l'OCDE. Ce type d'information existe aussi pour les appareils médicaux, pour les coefficients de dose, pour la gestion de certains accidents nucléaires, etc. Bref ce livre est très complet.

J.C. Amiard est très clair, il ne cache pas une certaine méfiance vis à vis du nucléaire, mais le fait sans exagération, ce qui fait qu'exprimer son point de vue ne se transforme pas en militantisme qui pourrait agacer et c'est tout à son honneur, et pour tout dire sa méfiance n'est pas immense.

J'ai toutefois sursauté une fois et souhaite que s'il y a une seconde édition, ce soit corrigé. Page 465 « la limite annuelle d'incorporation »? Pour avoir au sein du Comité 2 de la CIPR bataillé pour que cette notion disparaisse, puisqu'il n'y a qu'une limite pour le travailleur, la limite de dose efficace, accepter une limite annuelle d'incorporation serait accepter $20 \mathrm{mSv}$, pour un radionucléide, $20 \mathrm{mSv}$ pour un autre et ainsi de suite, on ne respecterait pas alors la réglementation. Je sais que cette notion avait ses adeptes et qu'elle rassurait certains mais il ne faut pas la présenter comme une limite, mais éventuellement comme un repère, un indicateur, mieux ne pas la présenter du tout. $2 / 3$ de page sur un tel ouvrage, avouez que la critique est limitée.

C'est pourquoi en conclusion et malgré son prix élevé (c'est une spécialité de Lavoisier), je recommande cet ouvrage qui peut servir à bon nombre d'enseignants, car les étudiants ne pourront pas s'offrir un tel ouvrage ... et à d'autres.

H. Métivier SFRP

DOI: 10.1051/radiopro/2013091

RADIOPROTECTION - VOL. 48 - N 4 (2013) 
REVUE DES LIVRES

La neutronique, Monographie de la Direction de l'énergie nucléaire du CEA, Le Moniteur, 2013, ISBN : 978-2-281-11371-6, 273 pages, $22 €$.

La neutronique c'est simple, c'est tout simplement l'étude du cheminement des neutrons dans la matière. C'est du moins ce qu'en dit la présentation de ce nouveau volume des monographies de la DEN que dirige notre ami Bernard Bonin.

Je suis d'accord avec cette définition, sauf que ce volume montre qu'il y a beaucoup plus et qu'il faut quand même un sacré bagage mathématique pour en être un acteur. La neutronique est née en 1932 et en France, elle est toujours très active, car à la base de la conception de tous les réacteurs nucléaires anciens, actuels et futurs, mais aussi lorsqu' on change tout simplement la nature du combustible $\mathrm{UO}_{2}$ en MOX. C'est ce qui est décrit dans cet ouvrage.

Cette monographie est très réussie, cela devient un pléonasme, car en plus de son contenu scientifique, elle est illustrée de nombreuses photographies et encarts de tous les acteurs de la neutronique, ce qui humanise grandement le propos (que du beau monde...). Ah quand je vois la photo de Harry Bateman, j'ai du mal à penser qu'il m'a tant fait souffrir avec ses problèmes de robinets et de baignoires qui fuient ou plus tard de filiations radioactives, mais c'est du passé !

Cette monographie complète une série déjà remarquable, quels seront les futurs numéros, nous les attendons déjà.

H. Métivier SFRP

Guide de la Radioprotection 2014, SNIIM, ISSN en cours, 161 pages.

Sous l'égide de leur syndicat national (SNIIM), les Ingénieurs de l'Industrie et des Mines, rédigent, depuis de nombreuses années, des guides sur leurs métiers exercés au sein des administrations de l'État touchant des domaines d'activité très variés. Dans ce cadre, ils viennent de publier un «Guide de la Radioprotection » pour faire bénéficier de leur expérience dans ce domaine qui s'est enrichie avec et au fur et à mesure de la construction du système français de contrôle de la radioprotection.

Les acteurs de la radioprotection, et en particulier les utilisateurs de rayonnements ionisants trouveront dans ce guide, des informations concrètes et pratiques à caractère administratif, réglementaire et technique. Ainsi le lecteur trouvera les réponses à ses questions dans les 11 chapitres du guide :

- Pourquoi la radioprotection

- Sources de rayonnements ionisants pour quelles utilisations

- Les acteurs de la radioprotection

- Le cadre réglementaire

- Les régimes administratifs

- La démarche à suivre pour exercer une activité nucléaire

- L'organisation «pratique » de la radioprotection

- Le transport de substances radioactives

- La gestion des déchets et des effluents radioactifs 
- L'organisation du contrôle de la radioprotection

- Les responsabilités civiles et pénales

Balayant l'ensemble de ces sujets en 161 pages, ce guide ne rentre pas dans les détails mais se présente comme un excellent mémento pour retrouver des informations de base. À noter aussi, la volonté d'y avoir inclus un répertoire des administrations et des professionnels du domaine de la radioprotection (ceci nécessitant néanmoins une certaine vigilance quant à l'exactitude des informations dont certaines sont déjà inexactes, les institutions changeant si rapidement).

Le guide contient également des fiches thématiques qui permettent de mieux appréhender, d'un point de vue pratique, les exigences réglementaires de certains domaines de la radioprotection. Il pourrait trouver tout naturellement sa place comme manuel de synthèse distribué dans certains enseignements de radioprotection.

Le rédacteur en chef précise qu'il s'agit d'une première version du guide qui comme les autres s'enrichira au fil des éditions successives.

V. Chambrette

Directrice de la SFRP

Physique, fonctionnement et sûreté des REP. Maîtrise des situations accidentelles du système réacteur, Bruno Tarride, EDP Sciences, 2013, ISBN : 978-2-7598-0738-3, 318 pages, $55 €$.

Les Réacteurs à Eau sous Pression (REP) ont pour fonction de convertir l'énergie libérée par les réactions de fissions nucléaires en énergie thermique véhiculée sous forme de vapeur, puis en énergie électrique. Ce procédé doit être particulièrement surveillé pour démontrer la capacité, en fonctionnement, à maîtriser la réaction en chaîne et, en toute circonstance, à assurer l'évacuation de la puissance thermique produite au sein du cœur du réacteur, ainsi que le confinement des matières radioactives.

Cet ouvrage aborde la gestion des situations accidentelles de la filière REP, et est composé de deux parties :

- l'une présentant les grandes familles d'accidents prises en compte dans la démonstration de sûreté (sûreté réglée), à savoir les accidents de réactivité par retrait d'absorbants ou surmodération des neutrons, les accidents mettant en cause un défaut d'extraction de la puissance du cœur, de son transport vers la source froide ou de disponibilité de cette source froide, enfin les accidents conduisant à un défaut du confinement ;

- l'autre présentant l'accident de référence de cette filière, l'accident de Three Mile Island, et ses enseignements pour développer la résilience du système (sûreté gérée), en particulier le développement d'une conduite post-accidentelle par Approche Par État et la gestion des accidents graves post-fusion du cœur.

De façon à améliorer la compréhension des divers transitoires présentés, des exercices et des problèmes sont proposés en fin de chapitres. En complément, le lecteur trouvera en annexe, une description des accidents de Tchernobyl et Fukushima (respectivement filières RBMK et REB), leurs principaux enseignements ainsi que les améliorations de sûreté induites 
REVUE DES LIVRES

sur le plan organisationnel et matériel. Sur ce dernier point, les principaux choix de conception du réacteur de troisième génération EPR sont présentés.

La spécificité de l'ouvrage est d'appréhender la dimension systémique du réacteur, en insistant sur les interactions entre sous-parties de la chaudière, les couplages physiques, les boucles de rétroaction... Une telle approche est indispensable aux ingénieurs du génie nucléaire pour analyser et anticiper le comportement du système réacteur en fonctionnement accidentel et ainsi pouvoir proposer des dispositions de conception ou d'exploitation afin d'en améliorer la sûreté.

L'air et l'eau. Alizés, cyclones, Gulf Stream, tsunamis et tant d'autres curiosités naturelles, René Moreau, EDP Sciences, 2013, ISBN : 978-2-7598-0828-1, 314 pages, $45 €$.

L'air et l'eau, chacun connaît ; nous savons que sans ces deux fluides, notre vie serait impossible sur Terre. Et après ? Que savons-nous sur eux ? Depuis quelques années, des événements graves se multiplient : cyclones, tornades, tsunamis, inondations... mais sait-on comment cela fonctionne?

Le livre apporte les réponses à ces questions. Tout d'abord, on considère l'air et l'eau dans des situations dites « à l'équilibre », c'est-à-dire pour lesquelles on se pose d'ordinaire peu de questions. Puis on aborde les phénomènes dynamiques aux diverses échelles. Le parti pris est de décrire avant d'expliquer. L'ouvrage se termine par la présentation de constructions humaines (barrages, ports...) destinées à domestiquer et utiliser l'eau à notre avantage.

Le lecteur se voit proposer une promenade à diverses échelles, des alizés aux nuages, du Gulf Stream aux vaguelettes. Ainsi il découvre et comprend de façon intuitive les phénomènes. Ceux qui désirent des démonstrations plus physiques et mathématiques iront visiter le site web en libre accès du pap-ebook et auront besoin d'un niveau scientifique supérieur. La majorité des lecteurs effectuera une promenade sur Terre avec nos deux fluides fétiches grâce à un livre remarquablement illustré avec ses index, glossaire, annexe, et aussi avec les films du site web. Ce livre restera un incontournable de la bibliothèque que l'on pourra conserver pour le consulter à nouveau, et l'utiliser comme base de toute recherche sur l'air et l'eau. 